\title{
The Genesis of Super Star Clusters as Self-Gravitating HII Regions
}

\author{
Jonathan C. Tan ${ }^{1}$ and Christopher F. McKee ${ }^{2}$ \\ 1 Dept. of Astronomy, UC Berkeley, Berkeley, CA 94720, USA \\ 2 Depts. of Physics and of Astronomy, UC Berkeley, Berkeley, CA 94720, USA
}

\begin{abstract}
We examine the effects of ionization, radiation pressure and main sequence winds from massive stars on self-gravitating, clumpy molecular clouds, thereby modeling the formation and pre-supernova feedback of massive star clusters. We find the process of "turbulent mass loading" is effective in confining HII regions. Extrapolating typical Galactic high-mass star forming regions to greater initial gas cloud masses and assuming steady star formation rates, we determine the timescales for cloud disruption. We find that a dense $\left(n_{c} \simeq 2 \times 10^{5} \mathrm{~cm}^{-3}\right)$ cloud with initial mass $M_{\mathrm{c}} \simeq 4 \times 10^{5} \mathrm{M}_{\odot}$ is able to form $\sim 2 \times 10^{5} \mathrm{M}_{\odot}$ of stars (50\% efficiency) before feedback disperses the gas after $\sim 3$ Myr. This mass and age are typical of young, optically visible super star clusters (SSCs). The high efficiency permits the creation of a bound stellar system.
\end{abstract}

\section{Introduction}

Most Galactic stars are born in highly clustered regions [12,5], where the disruptive effects of massive stars are paramount. The multitude of dusty high redshift sources and the intensity of the far infrared background they produce also imply that a major fraction $(\sim 1 / 2)$ of total cosmic star formation has occurred in starbursts, replete with massive stars [20]. A significant fraction of star formation in local starbursts occurs via the creation of super star clusters (SSCs) [22 24], each with hundreds to thousands of OB stars crammed into a few parsecs. At least some SSCs are gravitationally bound [8,9] and their masses $\left(\sim 10^{5}-10^{6} \mathrm{M}_{\odot}\right.$ [19, 15) and sizes suggest we may have found globular clusters in their infancy.

Massive stars violently disrupt their surroundings with ionizing and nonionizing photons, protostellar, main sequence and post-main sequence winds, and supernovae. Adding to this complexity is the extremely dense, clumpy and turbulent nature of the gas in which high-mass stars are born. We present a simplified model of pre-supernova feedback, to examine how the efficient star formation required to produce bound clusters may occur in the presence of vigorous energy injection from many massive stars.

\section{Initial Conditions - a Clumpy Molecular Cloud}

We consider a spherical cloud of radius, $R_{\mathrm{c}}$, mass $M_{\mathrm{c}}$, and mean $\mathrm{H}$ density $n_{\mathrm{c}}$. The cloud consists of dense clumps embedded in a uniform inter-clump medium. The clumps are distributed uniformly within a central core of radius $0.2 R_{\mathrm{c}}$, and with a $r^{-1}$ distribution outside, which mimics observed molecular cloud 
profiles. We choose mean clump mass and volume fractions of $f_{m}=0.8$ and $f_{V}=0.03$ respectively. Our clumps, of uniform density $n_{\mathrm{cl}}$, have a mass spectrum $\mathrm{d} \mathcal{N}_{\mathrm{cl}} / \mathrm{d} \ln m_{\mathrm{cl}} \propto m_{\mathrm{cl}}^{-0.6}$ between upper, $m_{\mathrm{cl}, \mathrm{u}}$, and lower, $m_{\mathrm{cl}, \mathrm{l}}$, limits. Clump masses span a range of $10^{3}$, with $m_{\mathrm{cl}, \mathrm{u}}=0.025 M_{\mathrm{c}}$, so no one clump dominates the cloud. The clump velocities are set so the cloud is in virial equilibrium.

Plume et al. 18 determined sizes and masses for 25 regions of Galactic highmass star formation. The mean properties of this sample were $R_{\mathrm{c}} \sim 0.5 \mathrm{pc}$, $M_{\mathrm{c}} \sim 3800 \mathrm{M}_{\odot}$ (virial mass) and thus $n_{\mathrm{c}} \simeq 2 \times 10^{5} \mathrm{~cm}^{-3}\left(\simeq 3.2 \times 10^{4} \mathrm{M}_{\odot} \mathrm{pc}^{-3}\right)$. Note that these regions are only a small fraction of the host Giant Molecular Cloud. Our adopted clump mass and volume fractions imply clump densities of $n_{\mathrm{cl}}=6.5 \times 10^{6} \mathrm{~cm}^{-3}$ and an inter-clump density of $5.0 \times 10^{4} \mathrm{~cm}^{-3}$. These properties form the basis of our model $\mathbf{A}$. Determining the gas properties of the precursors to SSCs is more difficult as current observations only probe scales down to $\sim 30-100 \mathrm{pc}[7,23]$, while the star clusters have a typical radius $\sim 4 \mathrm{pc}$ 22. For simplicity we consider models with $10(\mathbf{B})$ and $100(\mathbf{C})$ times the mass of $\mathbf{A}$, but with the same mean density. With the same values of $f_{V}$ and $f_{m}$ the clump and interclump densities are also the same. For each cloud we shall consider a fixed star formation rate, $\phi_{50}$, which converts $50 \%$ of the initial cloud into stars over $3 \mathrm{Myr}$ - i.e., the approximate time before the first supernova is expected to occur. The properties of our initial gas clouds are listed in table 1 .

Table 1. Model Parameters

\begin{tabular}{ccccc}
\hline Model & $M_{\mathrm{c}} /\left(\mathrm{M}_{\odot}\right)$ & $R_{\mathrm{c}} /(\mathrm{pc})$ & $\sigma_{1 \mathrm{D}} /\left(\mathrm{km} \mathrm{s}^{-1}\right)$ & $\phi_{50} /\left(\mathrm{M}_{\odot} \mathrm{yr}^{-1}\right)$ \\
\hline $\mathrm{A}$ & $4 \times 10^{3}$ & 0.5 & 2.8 & $5.8 \times 10^{-4}$ \\
$\mathrm{~B}$ & $4 \times 10^{4}$ & 1.1 & 5.9 & $6.6 \times 10^{-3}$ \\
$\mathrm{C}$ & $4 \times 10^{5}$ & 2.3 & 12.9 & $6.7 \times 10^{-2}$ \\
\hline
\end{tabular}

\section{$3 \quad$ Feedback Processes}

To model the feedback processes, we need the total number of hydrogen ionizing $(\lambda<912 \AA)$ photons emitted per second $(S)$, the bolometric luminosity $\left(L_{\text {bol }}\right)$ and the mass flux $\left(\dot{M}_{w}\right)$ and velocity $\left(v_{w}\right)$ of the stellar wind from the forming star cluster. We derive these quantities from the STARBURST99 model 13 at solar metallicity. As we are interested only in the first few Myr of stellar evolution, we approximate the values of these quantities to be constant for a given stellar mass. Motivated by observations of the stellar population of the R136 cluster in 30 Doradus 朋, we consider a stellar initial mass function (IMF)

\footnotetext{
${ }_{1}$ This rate excludes the formation of an initial star cluster of $\sim 250 \mathrm{M}_{\odot}$ necessary to produce an ionizing luminosity of $10^{49}$ ionizing photons per second (see below).
} 
represented by a broken power law, $\mathrm{d} \mathcal{N}_{*} / \mathrm{d} \ln m_{*} \propto\left(m_{*} / m_{*, 0}\right)^{-\alpha}$, with $\alpha=1.35$ (Salpeter) for $m_{*}>m_{*, 0}=3 \mathrm{M}_{\odot}$ and $\alpha=0.8$ for $m_{*}<m_{*, 0}$. We choose lower and upper limits of stellar mass of $0.1 \mathrm{M}_{\odot}$ and $100 \mathrm{M}_{\odot}$, respectively. This increases the mass per unit of feedback (assuming negligible contribution from stars below $\left.m_{*, 0}\right)$ by a factor of 1.35 compared to the standard STARBURST99 $\operatorname{IMF}\left(\alpha=1.35\right.$ between $1 \mathrm{M}_{\odot}$ and $\left.100 \mathrm{M}_{\odot}\right)$. We find

$$
\begin{gathered}
S_{49}=3.94 \times 10^{3} M_{*, 6}, \\
L_{\mathrm{bol}, 5}=1.10 \times 10^{4} M_{*, 6}, \\
\dot{M}_{w,-6}=3.09 \times 10^{3} M_{*, 6}, \\
\dot{M}_{w,-6} v_{2000}=3.98 \times 10^{3} M_{*, 6},
\end{gathered}
$$

where $S_{49}=S / 10^{49}$ photons $\mathrm{s}^{-1}, L_{\mathrm{bol}, 5}=L_{\mathrm{bol}} / 10^{5} \mathrm{~L}_{\odot}, \dot{M}_{w,-6}=\dot{M}_{w} / 10^{-6} \mathrm{M}_{\odot} \mathrm{yr}^{-1}$, $v_{w, 2000}=v_{w} / 2000 \mathrm{~km} \mathrm{~s}^{-1}$ and $M_{*, 6}=M_{*} / 10^{6} \mathrm{M}_{\odot}$. The number of $m_{*}>8 \mathrm{M}_{\odot}$ stars (i.e. number of core collapse supernovae) is $\mathcal{N}_{\mathrm{SN}}=1.4 \times 10^{4} M_{*, 6}$.

\subsection{Ionization}

Assuming spherical symmetry with the massive stars forming at the center of the cloud, we calculate the ionizing flux received at a given distance $R$ from the star cluster by accounting for attenuation by clump shadowing, $\mathrm{H}$ recombinations and dust absorption, so that

$$
\frac{d S}{d R}=-S \bar{A}_{\mathrm{cl}} \mathcal{N}_{\mathrm{cl}}-4 \pi R^{2} \alpha_{2} n_{i} n_{\mathrm{e}}-\sigma_{d} n_{i} S,
$$

where $\bar{A}_{\mathrm{cl}}$ is the mean cross sectional area of clumps at $R, \mathcal{N}_{\mathrm{cl}}$ is the space number density of clumps, $\alpha_{2}$ is the recombination coefficient, $n_{i}$ is the hydrogen nuclei number density of the ionized gas and $\sigma_{d}=0.5 \times 10^{-21} \mathrm{~cm}^{2}$ [1] is the dust absorption cross-section per $\mathrm{H}$ nucleus. We assume $\mathrm{He}$ is single ionized and $n_{\mathrm{He}}=0.1 n_{i}$. An HII region rapidly forms with typical size $R_{\mathrm{St}} \simeq 3.05 \times 10^{-2} S_{49}^{1 / 3} n_{i .5}^{-2 / 3} \mathrm{pc}$, for dust free, uniform density gas. The short sound crossing time justifies our assumption of uniform $n_{i}$. Thermal balance maintains a constant ionized gas temperature, $T_{i} \simeq 10^{4} \mathrm{~K}$. Over-pressurized compared to the surrounding neutral medium, the HII region tends to expand. . However, when neutral clumps become exposed to ionizing photons, they implode and inject mass into the HII region [2]. A compressed neutral globule remains, which continues to evaporate more slowly [3]. We employ models for magnetically supported clumps so that the pressure of the ionized gas streaming from the surface of an imploded clump at distance $R$ is

$$
\begin{aligned}
\frac{p_{\mathrm{c}}}{k} & \simeq 8.7 \times 10^{7} n_{\mathrm{cl}, 7}^{1 / 21}\left(\frac{S_{49}}{R_{\mathrm{pc}}^{2}}\right)^{4 / 7} m_{\mathrm{cl}}^{-4 / 21} \mathrm{~K} \mathrm{~cm}^{-3} \\
& =9.9 \times 10^{9} n_{\mathrm{cl}, 7}^{1 / 21}\left(\frac{M_{*, 6}}{R_{\mathrm{pc}}^{2}}\right)^{4 / 7} m_{\mathrm{cl}}^{-4 / 21} \mathrm{~K} \mathrm{~cm}^{-3},
\end{aligned}
$$

\footnotetext{
${ }^{2}$ For the propagation of ionization fronts into the clump and interclump material, we assume the neutral gas has a temperature of $80 \mathrm{~K}$.
} 
where $m_{\mathrm{cl}}$ is the clump mass in solar mass units and $n_{\mathrm{cl}}$ is the initial clump density. Clumps may only photoevaporate if this pressure is greater than the wind ram pressure (below). Evaporating clumps are gradually ejected from the HII region by the rocket effect. However, clumps orbiting in the cloud potential continue to enter the HII region and inject fresh gas. The HII region is limited by a recombination front as the ionized gas flows out at approximately its sound speed. We have examined the details of this mechanism of "turbulent mass loading" and its implications for Galactic ultracompact HII regions elsewhere [21.

\subsection{Winds}

Stellar winds carve out a hot, low density cavity at the center of the HII region. The wind's thermal pressure is suppressed by cooling caused by mass injected by photoevaporating clumps [17. Instead, we calculate the edge of the wind cavity by balancing the thermal pressure of the HII region with the wind ram pressure,

$$
\frac{p_{\mathrm{w}, \mathrm{ram}}}{k}=7.6 \times 10^{5} \frac{\dot{M}_{-6} v_{2000}}{R_{\mathrm{pc}}^{2}} \mathrm{~K} \mathrm{~cm}^{-3} \simeq 3.0 \times 10^{9} \frac{M_{*, 6}}{R_{\mathrm{pc}}^{2}} \mathrm{~K} \mathrm{~cm}^{-3} .
$$

In addition, this pressure contributes to the clump dynamics and may quench photoevaporation, particularly at small distances from very massive clusters. We treat photoevaporation mass injection into the wind via a supersonic mass-loaded wind model, e.g. [6]. In cases of extreme mass loading the ionizing photons may be trapped in the wind.

\subsection{Radiation Pressure}

Our fiducial clumps absorb most of the momentum flux of the star cluster's radiation. The pressure is

$$
\frac{p_{\mathrm{rad}}}{k}=8.7 \times 10^{9} \frac{M_{*, 6}}{R_{\mathrm{pc}}^{2}} \mathrm{~K} \mathrm{~cm}^{-3}
$$

\section{Numerical Method for Modeling SSC Formation}

With an N-body code, we follow the dynamics, masses and sizes of a collection of several thousand clumps, in the fixed potential of the initial cloud and subject to the feedback processes described above. At the same time we model the coupled evolution of a spherically symmetric wind cavity and HII region. While we do not account for clump collisions, we note that their initial mass spectrum is probably created by a steady-state balance of collisional fragmentation and agglomeration. Our model does yet include a physical mechanism for star formation and so we investigate the response of our fiducial gas clouds to different imposed steady star formation rates, such that $50 \%$ of the cloud would be converted to stars after 3 Myr. Stars are created from gas in the innermost clumps at these rates and 
artificially added to the central cluster. We start our models by instantly forming $\sim 250 \mathrm{M}_{\odot}$ of stars, equivalent to an ionizing luminosity of $S_{49}=1$. We examine the time taken for the HII region to reach $90 \%$ of the initial cloud radius, at which point star formation is assumed to cease. Photoionization gradually destroys our model clouds, A, B and $\mathbf{C}$, as shown in figure 1 .

\section{Discussion}

The turbulent, clumpy and self-gravitating nature of our clouds impedes their destruction and allows star formation to proceed to higher efficiencies. For example, an HII region in a uniform, quiescent cloud with the same mean density and initial star cluster as model $\mathbf{A}$, would destroy the cloud in $\sim 3 \times 10^{5}$ years, even with no additional star formation. However, with most of the cloud mass in dense clumps with velocities set by virial equilibrium, the process of "turbulent mass loading" confines the ionized gas for 1.5 - $2 \mathrm{Myr}$, even though by this time, five times the initial stellar mass has formed and feedback is correspondingly greater (model A). Reasonable models for clouds forming SSCs survive up to 3 Myr (models $\mathbf{B}$ and $\mathbf{C}$ ). The fraction of the HII region occupied by the wind cavity increases with star cluster mass.

After a few Myr our neglect of Wolf-Rayet winds and supernovae becomes important. Furthermore, our use of a fixed gravitational potential, our assump-

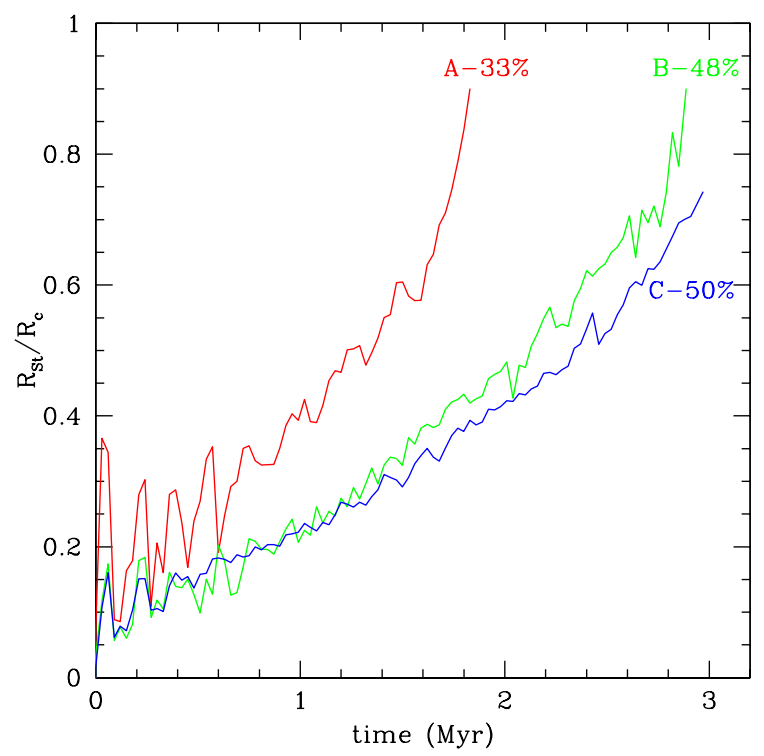

Fig. 1. Star cluster formation and cloud destruction at constant star formation rates, $\phi_{50} . \mathbf{A}: M_{c}=4000 \mathrm{M}_{\odot}\left(\right.$ Galactic case); B: $M_{c}=4 \times 10^{4} \mathrm{M}_{\odot} ; \mathbf{C}: M_{c}=4 \times 10^{5} \mathrm{M}_{\odot}$. Star formation efficiencies are quoted when $R_{\mathrm{St}}=0.9 R_{c}$ or after $3 \mathrm{Myr}$. 
tion that all massive stars are at the cloud center and the absence of protostellar winds in our model cause us to overestimate the cloud destruction time. Nevertheless, our results are qualitatively consistent with observed ages of the youngest optically visible SSCs, which are $\sim 5 \mathrm{Myr}$ 22]. Younger clusters are still embedded in dense gas [7,15]. R136 in 30 Doradus, which is considered a small SSC, has recently dispersed its gas and is $\sim 1-2$ Myr old 14.

With steady star formation rates over the cloud lifetime, our results imply that star formation efficiency increases with initial cloud mass. The high efficiencies apparent in our SSC models allow for the creation of bound clusters [1], even in the presence of vigorous feedback. Since the mass loss is gradual, efficiencies as low as $\sim 30 \%$ may result in loosely-bound clusters. Our Galactic model (A) is close to this limit, though the inclusion of additional feedback processes, such as protostellar winds, will reduce the efficiency.

We plan to extend our models to include additional feedback processes, such as protostellar winds, Wolf-Rayet winds and supernovae, and a physical star formation mechanism (e.g., photoionization regulated [16]) in the neutral clumps. We shall compare our models to observations of extra-galactic compact HII regions (e.g., [10]). We hope to predict minimum cloud masses and densities required to form bound stellar systems and probe in more detail the differences between Galactic and super star clusters.

\section{References}

1. J.A. Baldwin, et al.: ApJ, 374, 580 (1991)

2. F. Bertoldi: ApJ, 346, 735 (1989)

3. F. Bertoldi, C.F. Mckee: ApJ, 354, 529 (1990)

4. B. Brandl, et al.: In: From Darkness to Light, ed. by T. Montmerle, Ph. André (ASP conf. ser., 2000)

5. J.M. Carpenter: AJ, in press, (2000)

6. J.E. Dyson, R.J.R. Williams, M.P. Redman: MNRAS, 277, 700 (1995)

7. A.M. Gilbert, et al.: ApJL, 533, L57 (2000)

8. L.C. Ho, A.V. Filippenko: ApJL, 466, L83 (1996)

9. L.C. Ho, A.V. Filippenko: ApJ, 472, 600 (1996)

10. H.A. Kobulnicky, K.E. Johnson: ApJ, 527, 154 (1999)

11. C.J. Lada, M. Margulis, D. Dearborn: ApJ 285, 141 (1984)

12. E.A. Lada, K.M. Strom, P.C. Myers: 1993, In: Protostars and Planets III, ed. by E.H. Levy, J.I. Lunine (Univ. Arizona Press, Tucson 1993) p245

13. C. Leitherer, et al.: ApJS, 123, 3 (1999)

14. P. Massey, D.A. Hunter: ApJ. 493. 180 (1998)

15. S. Mengel, et al.: preprint, astro-ph/0010238 (2000)

16. C.F. McKee: ApJ, 345, 782 (1989)

17. C.F. McKee, D. Van Buren, B. Lazareff: ApJ, 278, L115 (1984)

18. R. Plume, et al.: ApJ, 476, 730 (1997)

19. A. Sternberg: ApJ, 506, 721 (1998)

20. J.C. Tan, J. Silk, C. Balland: ApJ, 522, 579 (1999)

21. J.C. Tan, C.F. McKee: in preparation (2001)

22. B.C. Whitmore, et al.: AJ, 118, 1551 (1999)

23. C.D. Wilson, N. Scoville, S.C. Madden, V. Charmandaris: ApJ, 542, 120 (2000)

24. Q. Zhang, S.M. Fall: ApJL, 527, L81 (1999) 